\title{
Laser-induced incandescence particle image velocimetry (LII-PIV) for two-phase flow velocity measurement
}

\author{
Luming Fan ${ }^{1}$ (D) Dante McGrath ${ }^{1} \cdot$ Cheng Tung Chong ${ }^{2} \cdot$ Mohammad Nazri Mohd Jaafar $^{3} \cdot$ Hongtao Zhong $^{4}$. \\ Simone Hochgreb ${ }^{1}$
}

Received: 6 July 2018 / Revised: 8 September 2018 / Accepted: 11 September 2018 / Published online: 1 October 2018

(C) The Author(s) 2018

\begin{abstract}
We demonstrate the use of laser-induced incandescence (LII) of submicron tungsten carbide (WC) particles as a method for particle image velocimetry (PIV). The technique allows a single laser to be used for separate measurements of velocity of two phases in a droplet-laden flow. Submicron WC particles are intentionally seeded into a two-phase flow, and heated by a light sheet generated by a double-pulsed PIV laser operating at sufficiently high pulse energy. The small size and large absorption cross section allows particles to be heated up to several thousand degrees Kelvin to emit strong incandescence signals, whilst the laser-induced temperature increase in liquid droplets/large particles is negligible. The incandescence signal from WC and Mie scattering from droplets/large particles are separately captured by deploying different filters to a PIV camera. The consecutive images of the laser-induced incandescence (LII) are used to determine the velocity field of the gas-phase flow, and those of Mie scatter are used to extract the velocity of droplets/large particles. The proposed technique is demonstrated in an air jet first and compared with the result given by a normal PIV test, which shows that submicron WC particles can accurately follow the gas flow, and that the LII images can be used to perform cross-correlations. We then apply this technique on an ethanol droplet/air jet (non-reacting), demonstrating the resulting slip velocity between two phases. The proposed technique combining PIV and LII with a single laser requires little additional equipment, and is applicable to a much higher droplet/particle density than previously feasible. Finally, the possibility of applying this technique to a flame is demonstrated and discussed.
\end{abstract}

\section{Introduction}

Two-phase flows are of wide interest for a variety of industrial processes, and especially for combustion conversion devices, such as liquid-fueled engines, gas turbines and solid fuel conversion. The interaction between fuel droplets/particulates and gaseous flows is of interest to capture

Luming Fan

lf368@cam.ac.uk

1 Hopkinson Laboratory, Department of Engineering, University of Cambridge, Trumpington Street, Cambridge CB2 1PZ, UK

2 China-UK Low Carbon College, Shanghai Jiao Tong University, 201306 Lingang, Shanghai, China

3 School of Mechanical Engineering, Faculty of Engineering, Universiti Teknologi Malaysia, 81310 Skudai, Johor, Malaysia

4 Department of Mechanical and Aerospace Engineering, Princeton University, Princeton, NJ 08544, USA the mass and heat transfer between particle and surrounding gas. Particle image velocimetry (PIV) is often used to collect information on the velocity field for each phase to validate models of droplet/particulate flow and reaction. However, such measurements are challenging for a number of reasons; in particular, droplet and particle sizes relevant to combustion applications range from tens to hundreds of micrometers. This has two consequences: (a) larger particles do not follow the gas flow, particularly under conditions where droplets are injected with high momentum relatively to the surrounding gas; (b) seeding micron-sized tracers into the two-phase flow cannot solve the problem, because the Mie-scatter PIV measurements produces blooming bright spots for the larger particles on the images over the much dimmer smaller particles, biasing and limiting the measurements under the limited dynamic range of typical cameras. There is a vast literature on developing advanced PIV techniques for multi-phase flow in non-reacting cases, but to the authors' knowledge, none of these studies were aimed at or conducted in reacting cases. Hence, the purpose of this 
study is to develop a new PIV technique that can be applied to measure both non-reacting and reacting two-phase flows. A summary and detailed classification of previous methods can be found in the work by Khalitov and Longmire (2002).

Methods for discriminating velocities in two different phases fall into two categories: color-based methods and Mie-scatter methods. These are described below in sequence.

Color-based methods typically use the fluorescence signal from micron-sized tracers doped with laser dye to separate Mie scattering from liquid droplets/non-fluorescent particles. The gas-phase velocity is derived from the former, whilst the velocity of the disperse form is extracted from the latter. Examples of this approach can be found in Towers et al. (1999); Driscoll et al. (2003). The technique suffers from the disadvantages that (a) fluorescent particles are relatively expensive or difficult to prepare in the laboratory, (b) the dyes used to dope the particles/droplets are usually highly toxic, requiring special safety procedures, (c) high laser energy is required for sufficient signal yield, and, most relevant to combustion studies, (d) the fluorescence signal disappears at high temperatures due to thermal quenching and decomposition of dyes in a flame. One of the most recent reports on fluorescence-based PIV by Petrosky et al. (2015) showed that KR620-doped particles could be a good surrogate for conventional fluorescence tracers due to their low toxicity. However, these particles do not overcome the problem of low signal yield.

Previous work Fan et al. (2016) demonstrated PIV measurements with phosphorescence signal using $\mathrm{ZnO}$ particles $(<2 \mu \mathrm{m})$ excited by a double-pulsed UV laser at $355 \mathrm{~nm}$, with an emission spectrum spanning from 350 to $450 \mathrm{~nm}$. Such a technique can be used for velocity measurements in two-phase flows under non-reacting conditions, using a suitable pulsed laser sheet in the UV range. Unlike specially made fluorescent particles, $\mathrm{ZnO}$ particles are inexpensive, non-toxic and the resulting light emission is sufficiently strong at the low temperatures, but its signal strength drops quickly as temperature increases, and can no longer be detected by unintensified CCD cameras beyond $500 \mathrm{~K}$.

An alternative approach to fluorescence and phosphorescence is based on the area dependence in Mie scattering: signal from both large droplets or solid particles and intentionally seeded micron-sized tracers are recorded by a single camera. Large particles are segregated from the smaller particles by preprocessing the PIV image before crosscorrelations are made. Sophisticated algorithms have been developed based on signal intensity differences (Sakakibara et al. 1996), spot size (Hassan et al. 1992), spot shape (Kiger and Pan 2000), or their combinations (Khalitov and Longmire 2002). The cost of the Mie-based method is relatively low on the experimental side, but the algorithms used are significantly more computationally intensive than in usual
PIV processing. Further, there is no universal algorithm for all two-phase flows, and image processing methods and filter parameters need to be selected based on the particular conditions of PIV images from each test. Factors such as the seeding density, contrast between large and small particles, and the background signals all have strong effects on the selection of filters and the sequence of operations. Experimentally, the limited camera dynamic range creates a problem: since Mie-scattering intensity is proportional to the square of particle size, apertures must be chosen so as to enable visualization of the smallest particles, yet the light scattered from particles of hundreds of micrometers size may exceed the exposure limit and permanently damage the CCD sensors. Finally, the main limitation of the Mie-based method is the requirement that the original liquid or solids particle concentration should be very sparse. Due to diffraction, particle images appear to be much larger than their physical size. Therefore, even for a moderate droplet/solid number concentrations in a gaseous flow, PIV images can be dominated by large bright spots, while the small and dim images of seeded tracers cannot be separated from those large spots. This strongly limits the application of Mie-based methods in combustion studies, because the spacing between fuel droplet/particles is designed to be sufficiently small to allow continuous flame propagation in the mixture, especially in the case of less volatile fuels (Myers and Lefebvre 1986). On the image processing side, difficulties include (a) decreases in droplet and particle size due to evaporation or reaction, (b) variable spatial concentration over sharp temperature gradients, and (c) image distortion near inhomogeneous gas refractive indices such as flame regions. To date, there has not been a general algorithm that can deal with all of these issues.

The absence of a velocity imaging technique for twophase flames has been reflected in recent combustion studies. In a recent review on work on spray combustion modeling (Sánchez et al. 2015), it was concluded that the slip motion of fuel droplets has an effect on the heat and mass transfer between the droplets and the gaseous flow. Most current studies on sprays rely on Laser Doppler Anemometry (LDA) to measure droplet velocities based on their size range by applying different masks, such as Kourmatzis et al. (2015) and Kariuki and Mastorakos (2017). However, LDA cannot resolve velocities spatially, and the velocity for different size ranges cannot be measured simultaneously. The difficulty in obtaining gas-phase velocity was also highlighted in a study on the combustion of aluminum suspensions (Julien et al. 2015). Julien and co-workers attempted to measure the burning velocity of aluminum particles $(\mathrm{SMD}=5.6 \mu \mathrm{m})$ on a counterflow burner with PIV, but they found that the slip motion of these particles was considerable. Since no technique was available to determine the fluid velocity directly from this particulate flame, they estimated the effective 
Stokes time by conducting a calibration in a cold impinging jet, and then corrected the lag in particle motion.

To implement velocity measurements in two-phase reacting flows, we propose using the laser-induced incandescence (LII) image pairs from submicron black tracers in PIV, rather than images of Mie scattering, fluorescence or phosphorescence. LII imaging traditionally works by heating soot particles to sufficiently high temperatures by a high-energy laser sheet so as to reach a fixed sublimation temperature, allowing the incandescence signal to be dependent primarily on the concentration of particles. A comprehensive review on the theory of LII and its application can be found in Michelsen et al. (2015). The LII technique has also been used to detect carbon black particles in dispersed form (Dankers et al. 2002). In addition, there have been several recent attempts to use LII technique as a diagnostic method for the flame synthesis of nano-particles such as silicon (Sipkens et al. 2013) and titania (Cignoli et al. 2009). In this study, submicron tungsten carbide (WC) particles were seeded into a droplet-laden flow and illuminated using a 532-nm PIV laser at a sufficiently high pulse energy. The WC submicron tracer particles were heated to several thousands of degrees to emit a strong incandescence signal, whereas the temperature increase for large droplets is negligible. The radiative incandescence due to high-temperature black body radiation can be collected away from the exciting wavelength (in this case, $400-450 \mathrm{~nm}$ ), thus allowing a separation of the signal from the submicron particles relatively to the liquid/solid phase.

This paper introduces the fundamentals of LII-based PIV, demonstrating the technique for non-reacting two-phase flows, and shows the possibility of application to reacting flows. The experimental setup for LII-PIV is described in Sect. 2. Section 3 demonstrates LII-based PIV in a singlephase air jet, and compares results with those obtained by Mie-based PIV with $1 \mu \mathrm{m}$ alumina particles. Velocity measurements in a non-reacting droplet-laden (ethanol SMD = $38.2 \mu \mathrm{m}$ ) flow and a methane/air flat flame are presented in Sects. 4 and 5, respectively.

\section{Experimental}

\subsection{Optical system}

A double-pulsed Litron Nano-PIV laser at $532 \mathrm{~nm}$ was used to generate a diverging light sheet with a thickness of approximately $0.3 \mathrm{~mm}$, by expanding the beam through a focal length $\mathrm{FL}=-150 \mathrm{~mm}$ concave cylindrical lens followed a FL $=500 \mathrm{~mm}$ convex lens. The height of the laser sheet above the test section is about $6 \mathrm{~cm}$. The laser energy used was $74.2 \mathrm{~mJ}$ for the first pulse, and $87.5 \mathrm{~mJ}$ for the second pulse, for an average laser fluence of 0.42 and $0.49 \mathrm{~J} /$ $\mathrm{cm}^{2}$, respectively. A $2048 \times 2048$ pixel CCD camera (Image Pro X 4M, LaVision) was operated in double frame mode at $5 \mathrm{~Hz}$ to collect both the LII and Mie-scattered signal. The exposure time for the first frame was fixed at $5 \mu \mathrm{s}$, and the exposure is fixed by the camera CCD technology to a minimum of $\sim 150 \mathrm{~ms}$ for the second frame. A Nikkor 60 $\mathrm{mm}$ micro lens was used to focus the image. The spatial resolution was $31.9 \mu \mathrm{m} / \mathrm{pixel}$ as calibrated by a target plate. An averaged background signal was recorded and subtracted from the 400 images collected. Due to a limited number of cameras available at the time of the test, the LII and Miescattering signal were captured non-simultaneously by the same camera, but with different filters: (a) for the LII signal, a $425 \pm 25 \mathrm{~nm}$ bandpass filter was installed in front of the lens and the aperture was opened wide $(f / 2.8 \mathrm{D})$ to guarantee a sufficiently strong LII signal; (b) for Mie scattering from micron-size particles, the combination of a ND $=0.6$ neutral density filter and $532 \pm 1.5 \mathrm{~nm}$ narrow bandpass filter was applied, and the lens aperture was set to f/5.6D; and (c) for the very strong scattering from large droplets, a ND = 2 filter was used instead, which reduces the signal to $1 \%$ of its original value, and the aperture was reduced to $f / 22 \mathrm{D}$ to avoid any damage to the CCD.

\subsection{Particles}

The tracer particle type for LII-PIV experiments in flames was chosen according to the following considerations: (a) the product of laser light absorption efficiency and energy used should be sufficiently large to obtain adequate LII signal strength; (b) the material should be non-reactive and able to withstand high temperatures; (c) the particle should be small enough to follow the sharp velocity gradients in a turbulent flame; and (d) the particles should be sufficiently large to produce a recognizable individual particle image for a successful cross-correlation. Ideal tracers for this technique should also be non-abrasive (for internal flow studies) and inexpensive (for large flow rate systems).

After considering a number of tracers, such as carbon black, graphite, manganese oxide and silicon carbide, the material selected was tungsten carbide (WC) with particles supplied by A.L.M.T. Corp. (WC02N). The Sauter Mean Diameter (SMD) of the WC particles is $0.2 \mu \mathrm{m}$, with a standard log mean deviation of $0.16 \mu \mathrm{m}$. The Stokes number of these particles is far below unity at the velocities considered; hence, their slip velocity should be negligible, as also demonstrated experimentally in Sect. 3. Tungsten carbide appears as black in powder form, which suggests that WC particles have a high absorption efficiency to visible and infrared light source. The melting point of WC is around $2800{ }^{\circ} \mathrm{C}$; so, particles remain stable at most flame temperatures. The WC powder is inexpensive $(<\$ 130 / \mathrm{kg})$ 
and non-toxic; so, it can be used in large quantities without safety concerns under well ventilation. These characteristics make WC particles an ideal candidate for the proposed LII-PIV technique. LII-PIV using carbon black and graphite was also attempted, but it was found to be more difficult to handle and disperse, and the results are not shown here. For comparison, a common PIV tracer, $1.0 \mu \mathrm{m}$ alumina (0CON007, Logitech Ltd.) was also applied to perform PIV measurements described in the following experimental sections.

\subsection{Seeding density}

Controlled seeding density is important for LII-PIV in a flame, as the flames may be sensitive to particle loading. Firstly, the local gas temperature may increase as the heat absorbed by particles from the light source is eventually passed to ambient gas via convection and radiation. This was reported for 2C-LII on a sooting ethylene diffusion flame (Nordström et al. 2014), where the soot volume fraction reported was several orders of magnitude higher than the normal seeding level for PIV measurements. In the present study, the particle concentration is estimated as $1.6 \times 10^{11}$ particles $/ \mathrm{m}^{3}$ based on the Mie-scattering images $(\sim 50$ particles per $32 \times 32$ interrogation window). With this seeding rate, the heating effect on local gas is estimated as follows.

Consider a single particle of diameter $d$ heated by uniform laser light of wavelength $\lambda$, with a fluence $F$ in the Rayleigh regime. The total energy absorbed is given by:

$q_{p}=\frac{4 \pi d E(m)}{\lambda} F \frac{\pi d^{2}}{4}$,

where $E(m)$ is the refractive-index function for absorption of the tracer particle. In the absence of data for WC particles, the value $E(m)=0.24$ for soot particles is assumed (Krishnan et al. 2001). For a wavelength of $532 \mathrm{~nm}$, a particle diameter of $200 \mathrm{~nm}$ and a mean fluence of $0.45 \mathrm{~J} / \mathrm{cm}^{2}$, the total energy addition is $1.6 \times 10^{-11} \mathrm{~J} /$ particle. For an estimated concentration of $1.6 \times 10^{11}$ particles $/ \mathrm{m}^{3}$, the total energy eventually transferred to the gas corresponds to $Q=2.6 \mathrm{~J} / \mathrm{m}^{3}$. The expected temperature rise for air properties of $\rho=1.2 \mathrm{~kg} / \mathrm{m}^{3}$ and $c_{p}=1000 \mathrm{~J} / \mathrm{kg} \mathrm{K}$ is therefore $\Delta T=\frac{Q}{\rho c_{p}} \approx \mathcal{O}\left(10^{-3}\right) \mathrm{K}$, which is clearly negligible.

A second consideration might be that the presence of particles with high radiative emissivity may increase the radiative heat loss from the flame: if the seeding density is too high, the temperature distribution in the flame may be changed; if too low, the particle images become too sparse to provide a velocity field with a decent spatial resolution. Here, an approximate calculation on the radiative heat loss is presented as follows. For a single spherical WC particle in a flame at $2100 \mathrm{~K}$, the radiative heat loss rate is estimated by the Stefan-Boltzmann law (Kock et al. 2006), and neglecting absorption of the radiation by surrounding particles, we have:

$\dot{q}_{r}=\pi d^{2} \varepsilon \sigma\left(T_{\mathrm{f}}^{4}-T_{a}^{4}\right)=1.4 \times 10^{-7} \mathrm{~W}$,

where $\varepsilon$ is the emissivity of tungsten carbide particles, taken as unity. $\sigma=5.67 \times 10^{-8} \mathrm{~W} /\left(\mathrm{m}^{2} \mathrm{~K}^{4}\right)$ is the Stefan-Boltzmann constant and $T_{\mathrm{f}}$ is the flame temperature. Considering a 20 $\mathrm{lpm}$ total gas flow rate with a seeding rate of $1.6 \times 10^{11} \mathrm{WC}$ particles $/ \mathrm{m}^{3}$, total radiative power produced by the glowing particles is

$\dot{Q}_{r}=\dot{q}_{r} N \approx 7 \mathrm{~W}$

For a gas mixture consisting of air/methane at stoichiometry, the heat release rate from combustion of the fuel is approximately

$\dot{Q}_{\mathrm{CH}_{4}}=1.2 \mathrm{~kW}$

This suggests that for usual PIV seeding levels (50 particles per interrogation window for the present configuration), the radiative heat loss by $\mathrm{WC}$ particles is negligible compared with the heat produced by the reaction.

\subsection{Effects of the LII decay time on the maximum measurable velocity}

In the present experiment, the tested flow speeds are lower than $15 \mathrm{~m} / \mathrm{s}$. Therefore, during the LII decay time (1-2 $\mu \mathrm{s}$, based on a preliminary test) the particle barely moves compared with the displacement during the pulse interval, so that particle images do not appear as streaks on any frames. Considering a decay time of $2 \mu \mathrm{s}, 31.9 \mu \mathrm{m}$ pixel resolution and a particle image size of 2-4 pixels, the maximum velocity allowed is estimated as $\sim 30 \mathrm{~m} / \mathrm{s}$ (during the decay time the particle moves less than 2 pixels whilst the signal strength decays exponentially). Beyond this velocity range, particle streaks may appear. However, in principle it should be possible to perform cross-correlations over particle streaks which start from a bright spot to a dark end, thus providing extra information for correlation peak detection. For supersonic applications, the LII signal would leave a long trajectory ( $>20$ pixels for current setup) on the images. Direct correlation on these trajectories may not be possible with the proposed technique. In this scenario, further image processing may be required to capture the starting location of particles, which is beyond the scope of this paper. 


\section{LII-PIV demonstration on an air jet}

\subsection{Operating conditions}

The proposed technique was first tested on a seeded air jet of diameter $11.7 \mathrm{~mm}$ at a flow rate of $50 \mathrm{slpm}$, corresponding to a bulk velocity around $8.5 \mathrm{~m} / \mathrm{s}$ at $298 \mathrm{~K}$ and a Reynolds number $R e=6600$. The air flow rate was metered by a 250 slpm Alicat Mass Flow Controller (MFC), with 1\% full scale precision. The time interval between two laser pulses was set to $\delta t=40 \mu \mathrm{s}$. Tungsten carbide particles $(0.2 \mu \mathrm{m})$ and fine alumina particles $(1 \mu \mathrm{m})$ were seeded to the air jet separately by a seeder (Texas Airsonics AJ-1), and measurements were made based on the Mie and LII signal, respectively. Before loading new tracers, the seeder, pipe and jet were flushed by 200 slpm air flow rate for $20 \mathrm{~min}$ to ensure that there were no residual particles in the flow system. Figure 1 shows the PIV images recorded at the green (532 nm, top row) and blue (425 $\mathrm{nm}$, bottom row) channels for the two types of particles. The absorption cross section of white alumina particles is much smaller than that of tungsten carbide; hence, no LII signal can be detected from alumina tracers, as shown in Fig. 1 ( bottom row). Note that the laser fluence used in this study is too low to heat particles larger than several micrometers to LII temperatures, even if they have large absorption efficiency (black), as also determined in a study of coal particles (Balusamy et al. 2015). Hence, this technique can potentially be applied to measure two-phase flows laden with large coal or metal particles, as the latter will not produce detectable LII signals.

\subsection{Raw images and data processing}

Figure $2 \mathrm{a}, \mathrm{b}$ show the zoomed-in view of a Mie-scatter and a LII image extracted from the first frame, both based on the WC tracer. As mentioned earlier, for the velocity in this case, the particle displacement during this signal decay time is less than 1 pixel, which cannot be resolved by the camera. Hence, particle images do not appear as streaks on any frames.

The Mie-scatter images in Fig. 2a shows clear particle images on a clean background, whereas the LII image, Fig. $2 b$ shows blurred particle images on a background of $\sim 30$ counts. The mean intensity of LII spots is about 200 counts (maximum 800 counts) for a noise level of 5 counts, whilst the mean intensity of Mie-scatter particle image is around 300 counts. According to the LII theory, the incandescence signal strength is approximately proportional to $T_{p}^{4}$, and the particle temperature $T_{p}$ depends on the local laser fluence $F$. The laser sheet used in this experiment had

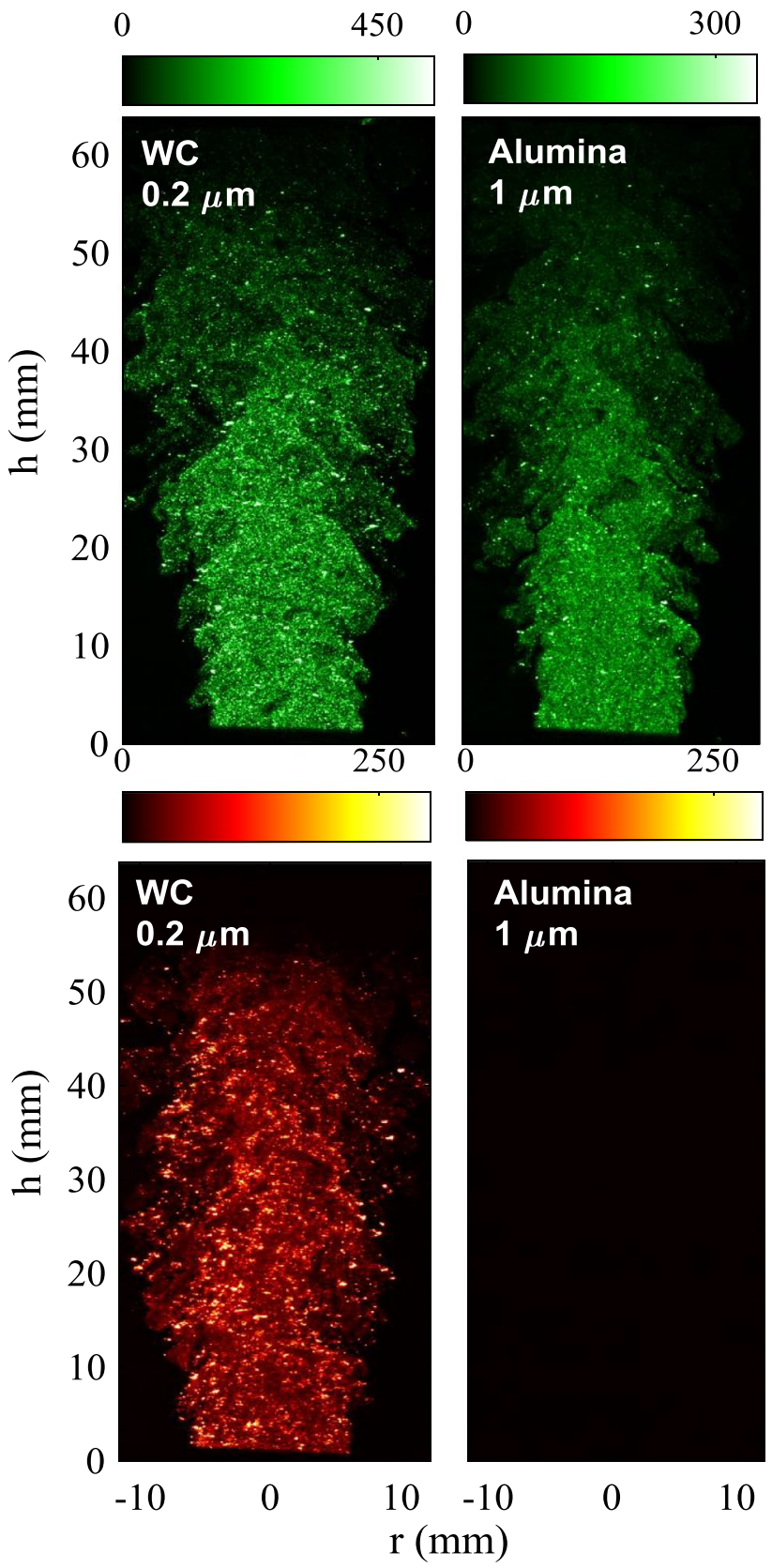

Fig. 1 First frame shots of a PIV image pair based on Mie-scattering (top row), and LII signal (bottom row) in an air jet. Tracers are $0.2 \mu \mathrm{m}$ WC and $1 \mu \mathrm{m}$ alumina, respectively (columns). The colorbars show the actual intensity counts recorded by the camera, which saturates at 9900 counts and has a dark noise around 5 counts. Note that the hot colormap is used to represent the incandescence signal, but it was actually collected from a blue channel $(425 \pm 25 \mathrm{~nm})$

a Gaussian profile along the out of plane direction. Hence, the brightest spots may be particles located close to the peak of the Gaussian profile, whilst particles subjected to the wings of the light sheet only produce very weak spots or form a vague background when projected onto the image plane as their temperature is much lower. This may 
Fig. 2 a Mie-scatter image of WC particles; $\mathbf{b}$ LII image of WC particles, both extracted from the first frame; c preprocessed LII image; $\mathbf{d}$ velocity field obtained at the same position based on the LII image pair (second frame not shown here). To present the robustness of the proposed technique, the vector field shown in $\mathbf{d}$ has not been post-processed by any interpolation or smoothing, and the interrogation region for removed vectors are shown in white. The area of the shown region is 200 $\times 200$ pixel $\left(6.4 \times 6.4 \mathrm{~mm}^{2}\right)$

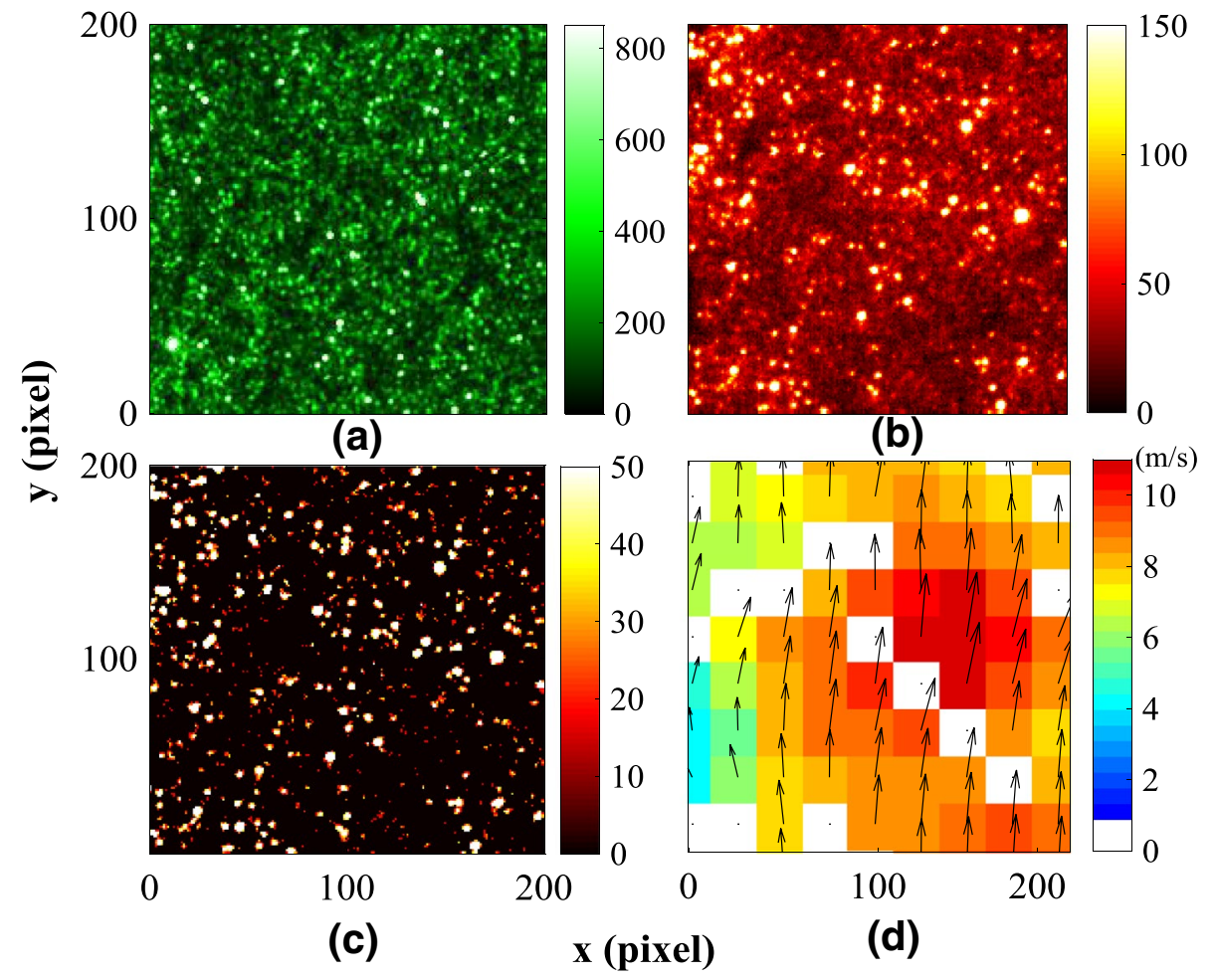

vectors. To show the robustness of LII-based PIV, the removed vectors were left blank without interpolation and no further post-processing was applied. An example of the raw vector field is shown in Fig. 2 d.

\subsection{Results and discussion}

Figure 3 shows an example of a single-shot velocity field measured by Mie-scatter and LII signal, respectively. In both cases, the cross-correlation produces quite a few spurious vectors, which have been removed during postprocessing. This is not uncommon for PIV measurements on turbulent flows, as the interrogation window size (of up to $\sim 1 \mathrm{~mm}$ ) is much larger than the Kolmogorov scale, and particles within one window may move at different velocities, thus producing a weak correlation peak. The averaged percentage of remaining vectors in the marked region over the whole 400 single-shots is calculated as $87 \%$ and $84 \%$ for Mie-scatter and LII-based PIV, respectively. This is an acceptable level for a turbulent jet of $R e=6600$. By that metric, the experiments show that LII technique is as good as traditional Mie-scatter PIV to measure single-shot velocity fields without losing too much spatial information in the core region, even though the number density of LII particle images is relatively lower than those of Mie scatter after preprocessing. The vectors on the top region of Fig. $3 b$ are missing due to low signal to noise ratio, as the particles here are approaching the edge of the light sheet where the laser fluence drops quickly. This occurs 
Fig. 3 Example of single-shot velocity field based on a Mie scattering of $1 \mu \mathrm{m}$ alumina and b LII signal from WC. Note they were taken non-simultaneously. Removed vectors are intentionally left blank rather than interpolated. The percentage of remaining vectors in the marked region after median filtering (averaged over 400 single-shots) is $87 \%$ and $84 \%$ for Mie-scatter and LII-based PIV, respectively
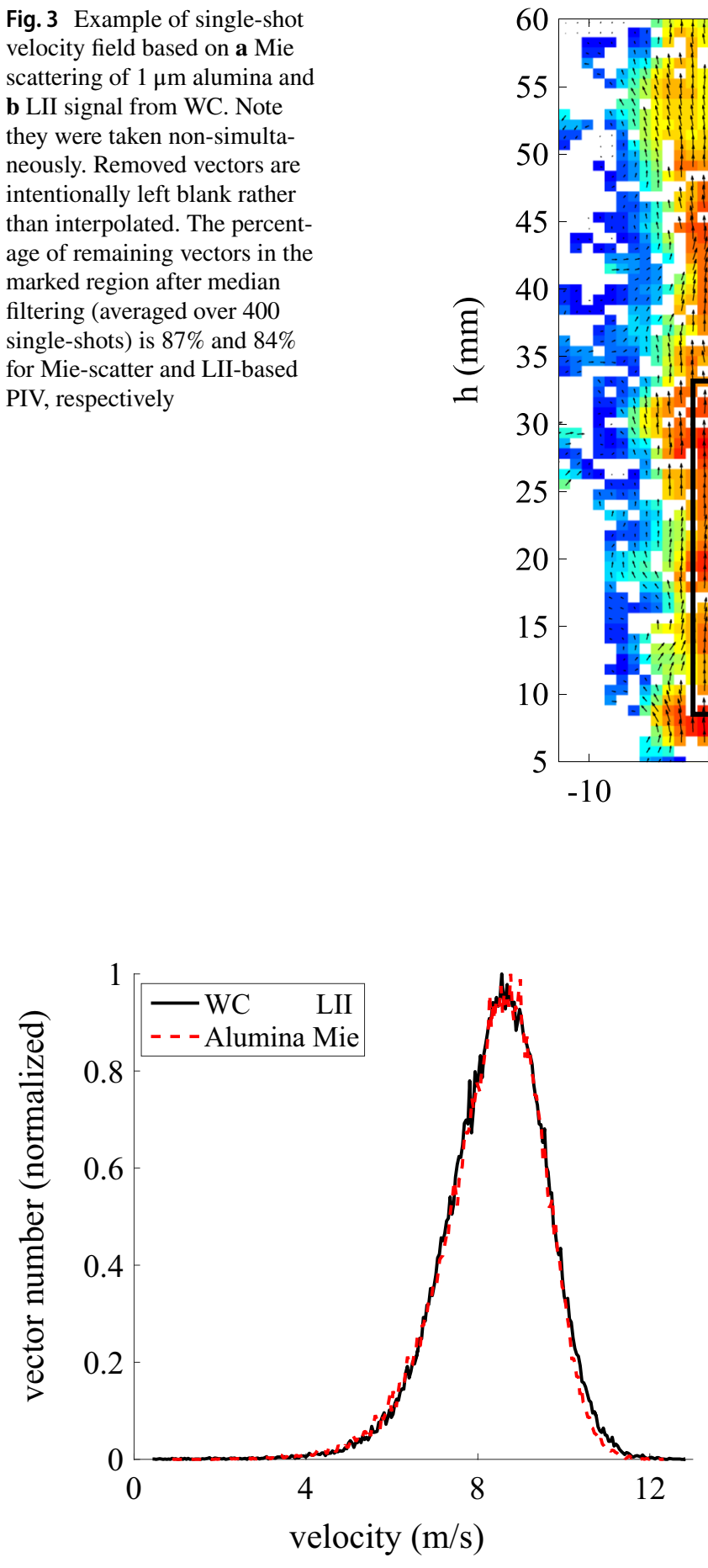

Fig. 4 Velocity probability density function (normalized) calculated from the marked region in Fig. 3 over 400 single-shots. Black line denotes the LII-based PIV, and red dotted line represents the result from Mie-scatter PIV

because the LII-PIV is much more sensitive to the local laser fluence than Mie-scatter PIV, suggesting a potential limitation of the technique. The velocity probability density function (PDF) in the same region is plotted in Fig. 4.
The velocity PDF extracted from the LII images matches well with that from the Mie-scatter PIV images.

Figures 5a and 6a show the mean velocity $\langle U\rangle$ and its rms $\langle u\rangle$ measured with the two types of tracers, namely $1 \mu \mathrm{m}$ alumina and submicron WC. The velocity profiles at $h=15,25$, and $35 \mathrm{~mm}$ are extracted and compared in Figs. $5 \mathrm{~b}$ and $6 \mathrm{~b}$. As shown in Fig. 5, the LII-PIV with WC agrees well with the results given by the $1 \mu \mathrm{m}$ alumina case (normal PIV), which shows that the selected submicron WC tracer can accurately follow the flow, and velocities can be accurately extracted from LII image pairs. The velocity rms in the shear layer obtained from LII signal is slightly higher than the normal PIV result, shown in Fig. 6. Again, this is probably due to the entrainment of unseeded air, leading to a poorer signal to noise ratio at this location for LII images. Adding a seeded coflow with WC particles can solve this problem.

\section{LII-PIV on a non-reacting droplet-laden flow}

\subsection{Operating conditions}

In this section, LII-based PIV was applied to measure the velocity field for the gas phase, while Mie-scatter PIV is used for the liquid phase in a droplet-laden flow. A Delavan AL-06 air-assisted atomizer was used to generate 
Fig. 5 a Mean velocity field for the same air jet, measured with $1 \mu \mathrm{m}$ alumina (left half) and $0.2 \mu \mathrm{m}$ WC (right half). b The velocity profiles at $h=15,25$, $35 \mathrm{~mm}$ are extracted and compared. The LII-PIV with WC matches well with the results given by the $1 \mu \mathrm{m}$ alumina case (normal PIV)

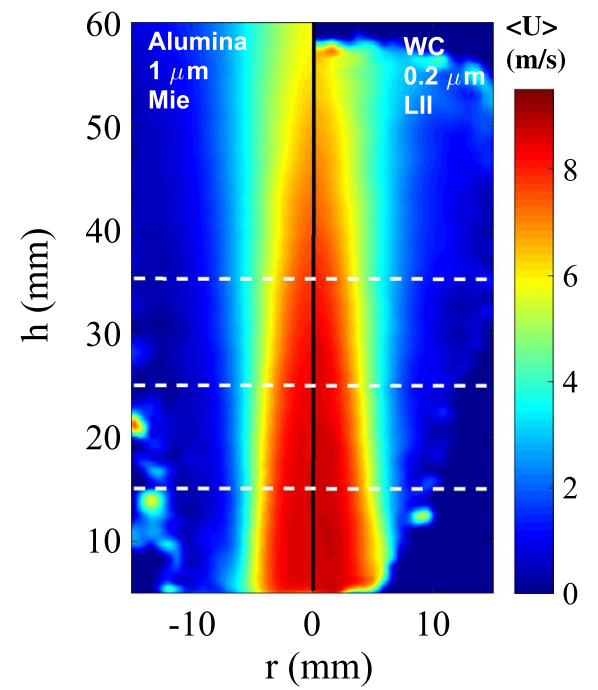

(a)

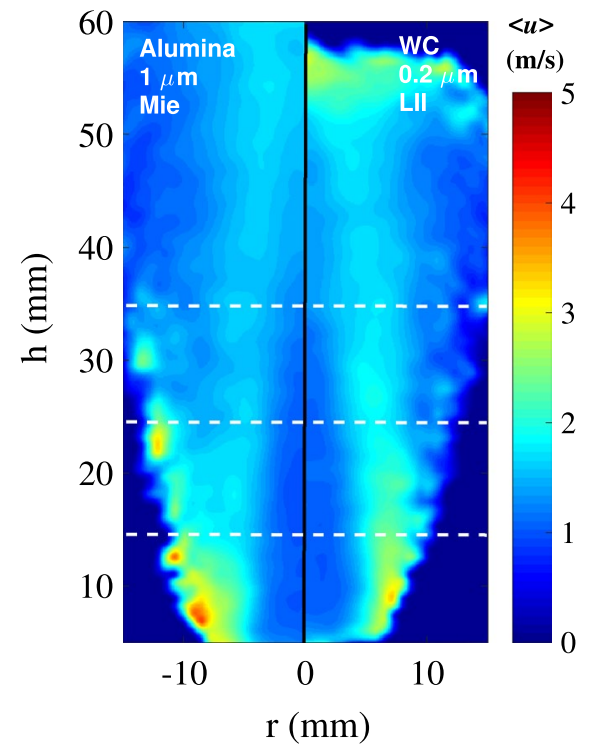

(a)

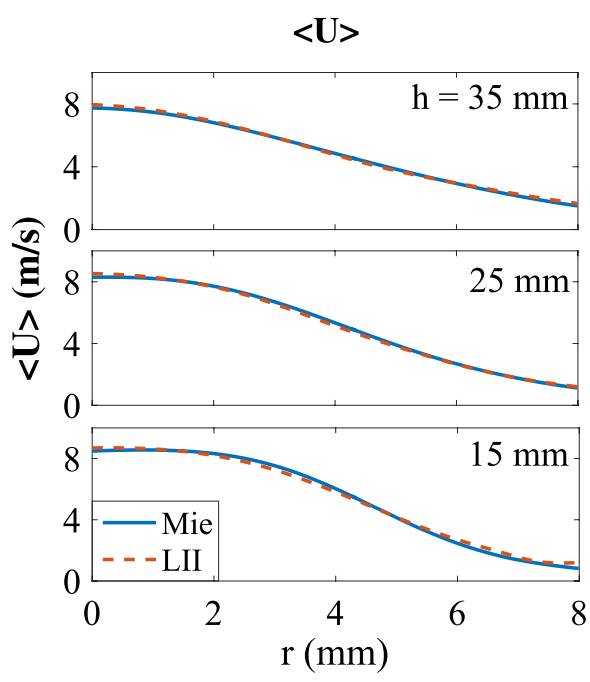

(b)

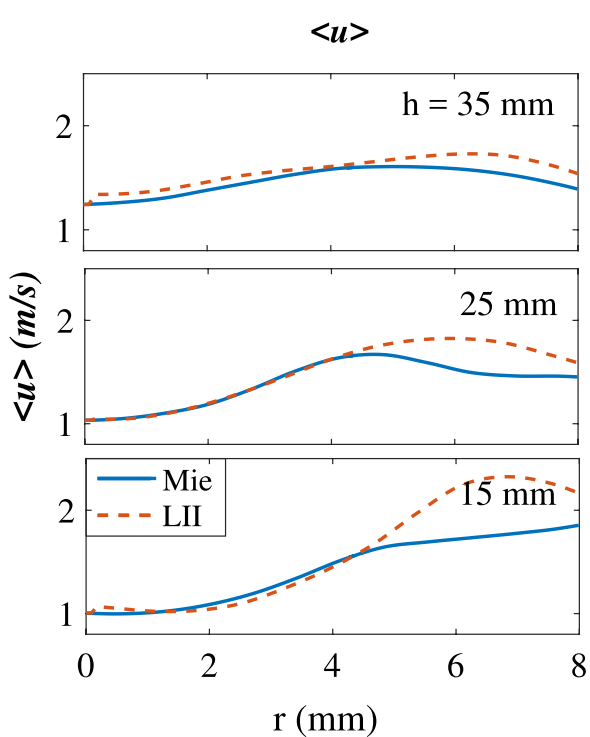

(b) ethanol droplets. Ethanol was delivered into the atomizer by a syringe pump, and the liquid flow rate was calibrated as $6 \mathrm{ml} / \mathrm{min}$ by a micro-scale. The air flow rate through the atomizer was $5 \mathrm{slpm}$, metered by a $20 \mathrm{slpm}$ Alicat MFC. The atomizer was installed into the bottom of a laminar flame burner, shown in Fig. 7a schematically. The burner consists of a 90-mm-diameter chamber, with a height of $260 \mathrm{~mm}$ which converges to an aerodynamically shaped nozzle of 22-mm exit diameter shrouded by a 5-mm-wide annular co-flow. For this non-reacting case, no co-flow was used to shield the droplet-laden flow. In spite of the high instantaneous temperatures reached by the WC particles, no ignition was observed. The measured droplet SMD was $38.2 \mu \mathrm{m}$ and the size distribution PDF is shown in Fig. 7b, which was measured by a Dantec PDA system at the outlet of the burner. WC tracers were carried by Air 2 (35 slpm, controlled by a 50 slpm Alicat MFC), which was mixed with the liquid droplets. The bulk velocity at the burner outlet was about $1.9 \mathrm{~m} / \mathrm{s}$ at room temperature and $R e=$ 2800. The time interval between two laser pulses was set to $230 \mu$ s for this case. The atomizer itself also generated some acoustic noise and turbulence to the droplet-laden flow. Mie-scatter and LII signals from the droplet/WC/air mixture were recorded separately using the same PIV camera. As mentioned in Sect. 2.1, a ND = 2 filter and a small aperture $(f / 22 \mathrm{D})$ were applied to protect the CCD from overexposure when capturing the Mie-scattering signal. 


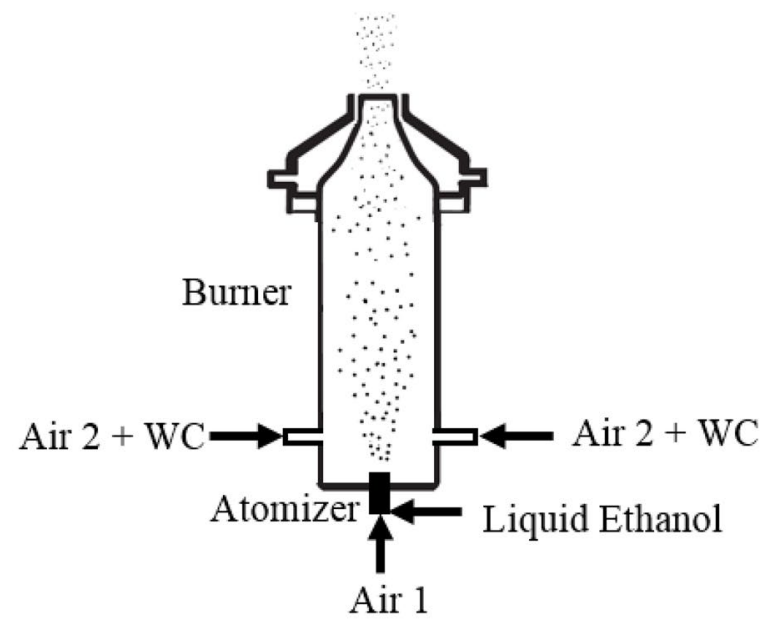

(a)

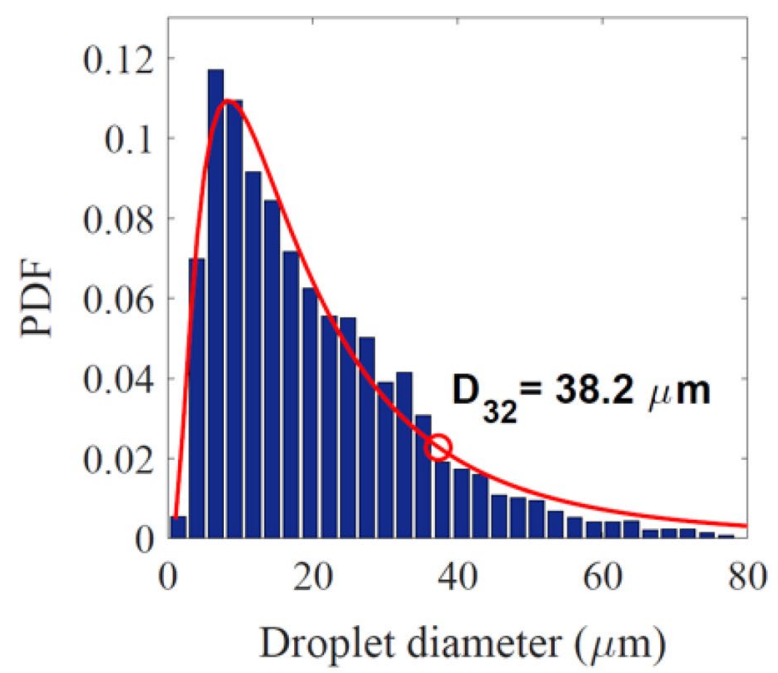

(b)

Fig. 7 a Schematic view of the laminar flame burner and the dropletladen flow; $\mathbf{b}$ size distribution of the ethanol droplets measured by a PDA system at the outlet of the burner. A total number of 5000 droplets were sampled, and the size distribution is cut off at $80 \mu \mathrm{m}$, limited by the mask installed in front of the PDA detector. The bars show the measured data and the red curve is the best log-normal fitting to the bars

In this configuration, the Mie scatter from the $0.2 \mu \mathrm{m} \mathrm{WC}$ particles cannot be detected, as it is lower than the signal scattered from the much larger ethanol droplets by several orders of magnitude. Therefore, Mie scattering from liquid droplets and LII signal from WC can be captured separately to determine the velocity field for each phase. After the two-phase PIV measurement, a conventional PIV test with $1 \mu \mathrm{m}$ alumina particles was conducted for comparison without the presence of ethanol droplets.

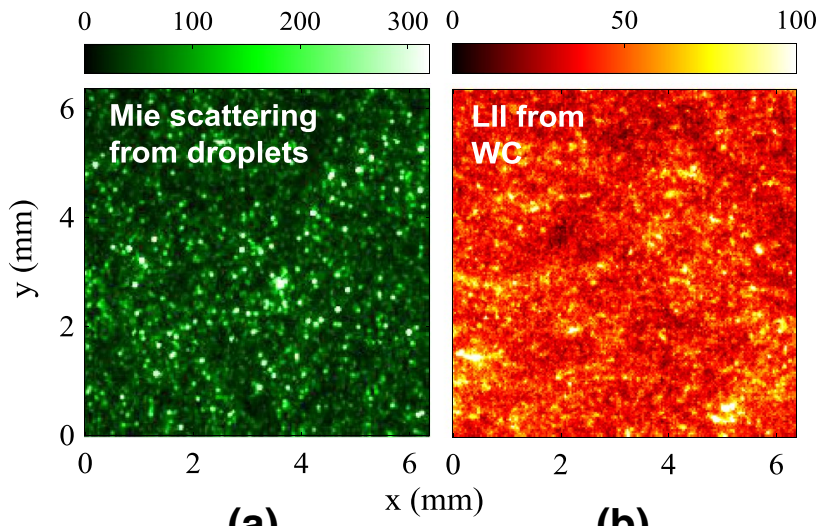

(a)

(b)

Fig. 8 Zoomed-in view of a Mie scattering and $\mathbf{b}$ LII image from the droplet/WC/air mixture

\subsection{Results and discussion: LII and droplet PIV}

Figure 8 shows sample images of the liquid droplet (Mie) and WC particles (LII) taken from the droplet/WC/air mixture. The velocity of droplets was extracted from the former, and the gas-phase velocity from the latter. Unlike Fig. $2 b$, the LII particles image in Fig. $8 \mathrm{~b}$ are smeared, which is probably due to multiple scattering of the LII signal in the dense droplet cloud. However, this does not affect the cross-correlation, as shown later on. Note that it would not be possible to apply any Mie-based methods to separate the gas-phase and droplet velocity for the high droplet density shown in Fig. 8a.

During the mixing process in the chamber, some WC particles may be captured by the liquid, as we noticed after the experiment that some WC tracers were accumulated on the sidewall and at the bottom of the burner. This was not found for the case without the presence of droplets. It suggests that (a) for an extremely high droplet number density, and thus a narrow interdroplet spacing, solid tracer-based twophase PIV may not be applicable as most particles will be captured by liquid, and the remaining free particles may be too sparse for conducting a successful cross-correlation; and (b) frequent cleaning may be necessary if the measurement is conducted in a confined space with sprays, such as in a chamber or cylinder. However, we note that the same issue would be present for the use of fluorescent or phosphorescent dyes on tracer particles.

The same data processing procedure described in Sect. 3.2 was applied to process the PIV images and to calculate the vector field. Figure 9 shows the velocity field based on Mie scattering from the droplets and LII from WC particles. The former represents the velocity for the disperse phase, and the latter for the continuous phase.

Figures 10 and 11 show the mean and rms velocity measured by droplets (Mie scattering) and WC particles (LII). The mean velocity fields given by the Mie signal and 
Fig. 9 Single-shot velocity field based on a Mie scattering from droplets and b LII signal from WC, which represents the gasphase motion in this dropletladen flow. Removed vectors are left blank intentionally. Note they were taken non-simultaneously

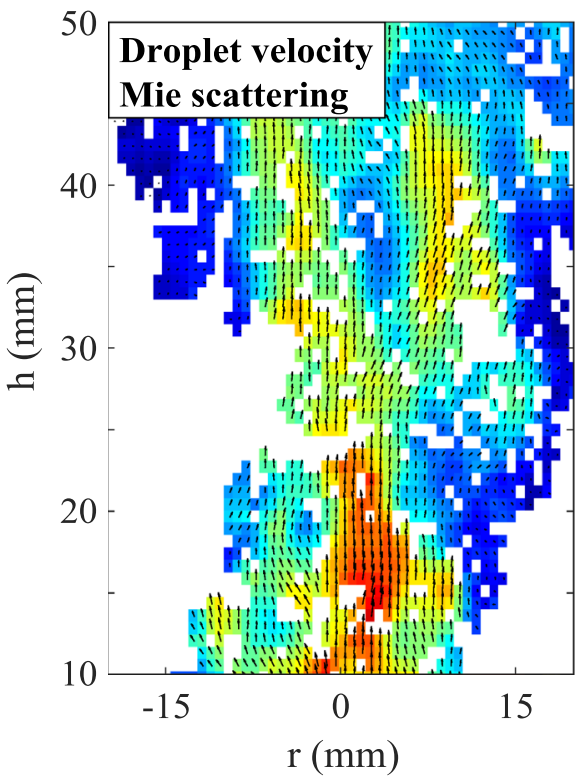

(a)

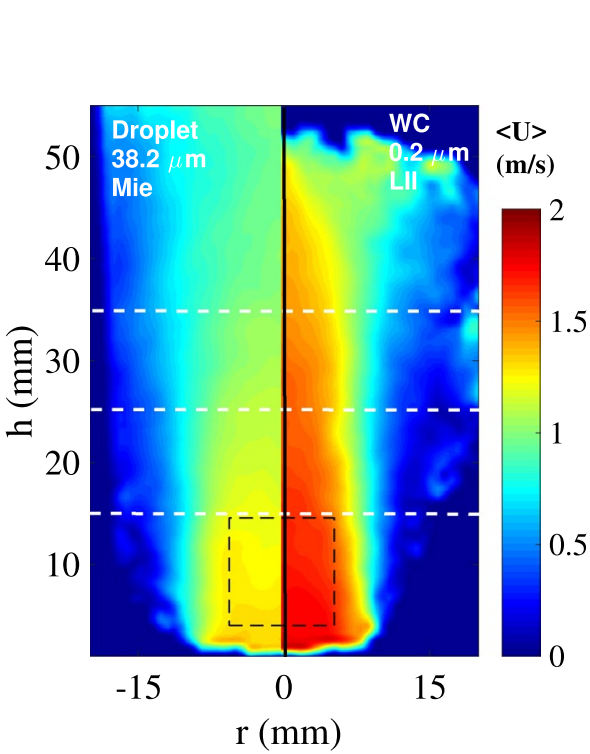

(a)

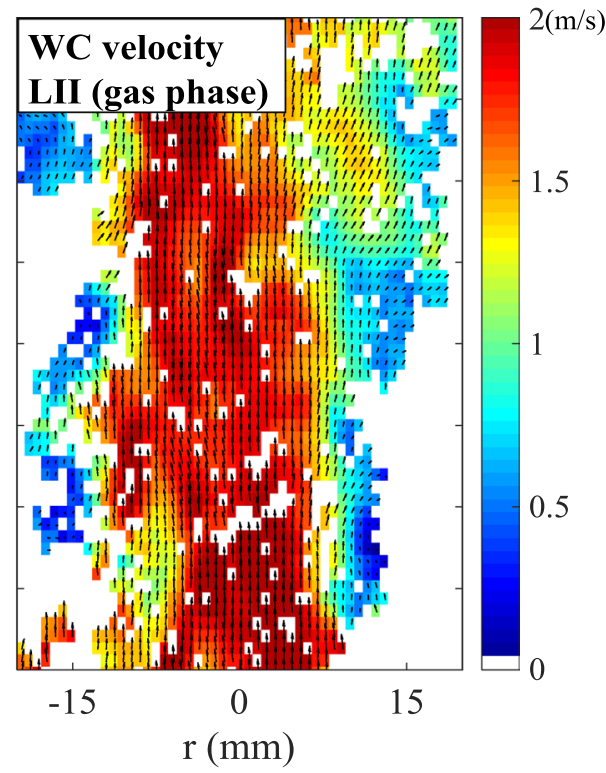

(b)
Fig. 10 a Mean velocity field of liquid phase (left) and gas phase (right); $\mathbf{b}$ the velocity profiles at $h=15,25,35 \mathrm{~mm}$ are extracted and compared. Ethanol droplets move significantly more slowly than the gaseous flow due to the effect of gravity relatively to the drag force. The dashed square marks the region where velocity PDFs in Fig. 12 are calculated

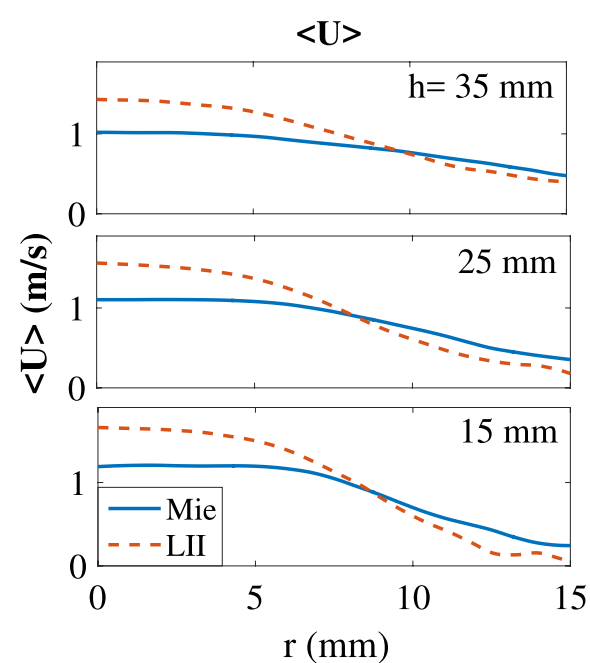

(b)
LII signal are very different, as shown in Fig. 10a, b. This could be attributed to the inertia of liquid droplets, which move significantly slower than the gas. Gravity also plays a role, as it could be verified by the observation that some droplets start to move downwards at around $10 \mathrm{~cm}$ above the nozzle. A quantitative comparison is made by comparing the velocity PDFs in the core region, and presented in Fig. 12. The slip velocity between droplets and gas phase can be clearly observed from the PDFs. Interestingly, the gas phase velocity measured in the presence of droplets (black line) shows a slightly wider distribution than that with no droplets (red dotted line). This implies that the large droplets may interact with the gaseous flow, and lead to a higher fluctuation in the gas-phase velocity.

\section{Demonstration of LII-PIV on a flame}

\subsection{Operating conditions}

In this section, the LII-PIV technique was demonstrated on a canonical stagnation flame using the same laminar flame burner, shown in Fig. 13. The atomizer was removed from the burner and the hole was sealed. A water-cooled brass 
Fig. 11 a Velocity rms of liquid phase (left) and gas phase (right); $\mathbf{b}$ the velocity profiles at $h=15,25,35 \mathrm{~mm}$ are extracted and compared. In the jet core (well seeding) the gas-phase velocity fluctuation is slightly higher than that of the liquidphase

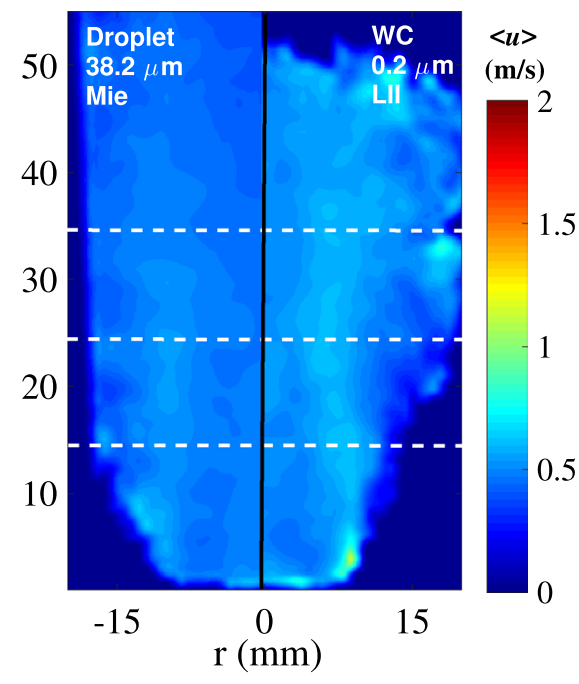

(a)

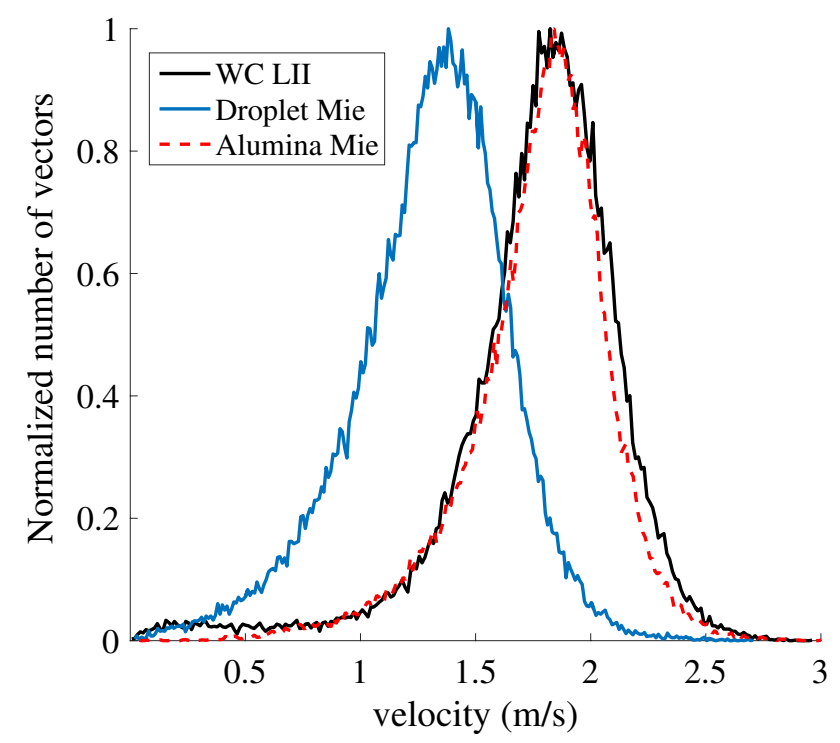

Fig. 12 Velocity PDFs calculated over the marked region in Fig. 10a. Black line shows the gas phase velocity, whilst blue line presents the droplet velocity. For comparison, the velocity PDF based on $1 \mu \mathrm{m}$ alumina (red dotted line) measured without the presence of droplets is also plotted

plate was placed over the nozzle of the burner, and the distance between the plate and nozzle was fixed at $25 \mathrm{~mm}$. Methane was used as the gaseous fuel with a flow rate of 2.7 slpm, metered by a 20 slpm Alicat MFC. The air flow rate was set to $30 \mathrm{slpm}$, leading to an equivalence ratio of $\phi$ $=0.86$. An unseeded nitrogen co-flow was controlled by a 250 slpm Alicat MFC to provide the same co-flow velocity as the main flow. A flat flame is stabilized $5 \mathrm{~mm}$ beneath the surface of the cooling plate. It can be observed from Fig. 13 that the flame luminosity is very strong due to radiation

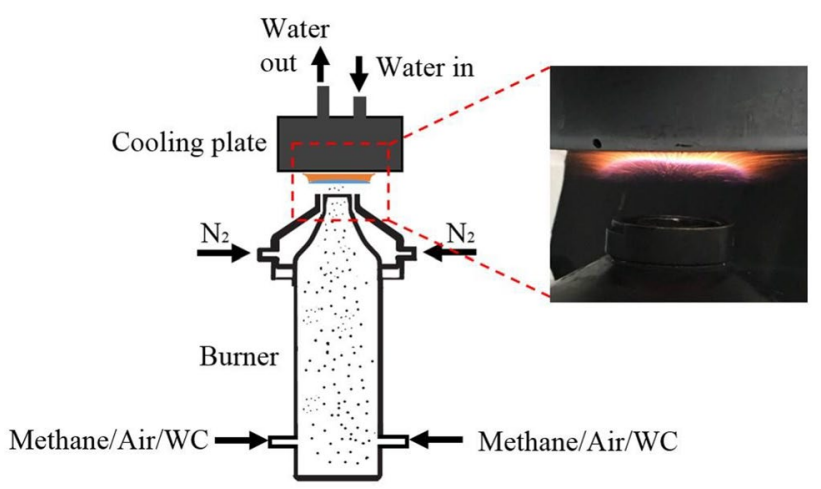

Fig. 13 Schematic view of the stagnation flame configuration

from the seeded black WC particles; this saturates the second frame of the LII image and causes a problem for the cross-correlation in the post-flame region, as described later. Tracer particles of diameter $1 \mu \mathrm{m}$ alumina (normal PIV) and $0.2 \mu \mathrm{m}$ WC were used for Mie-scatter PIV on this reacting cases separately. For this laminar flame case, 200 images were recorded for each dataset, and a single pass of $32 \times 32$ pixel interrogation window with $25 \%$ overlap was applied to calculate the vector field.

\subsection{Results and discussion: WC flame PIV}

Figure 14 shows the result of PIV for the stagnation flame. Figure 14a, b are sample single shot first frames of Mie scatter (left) and LII (right), respectively. The particle density drops by a factor of around seven downstream of the flame front, due to thermal expansion of the flow (thus a much lower seeding density) and the out-of-plane motion of tracer particles as they are approaching towards the cooling plate. 


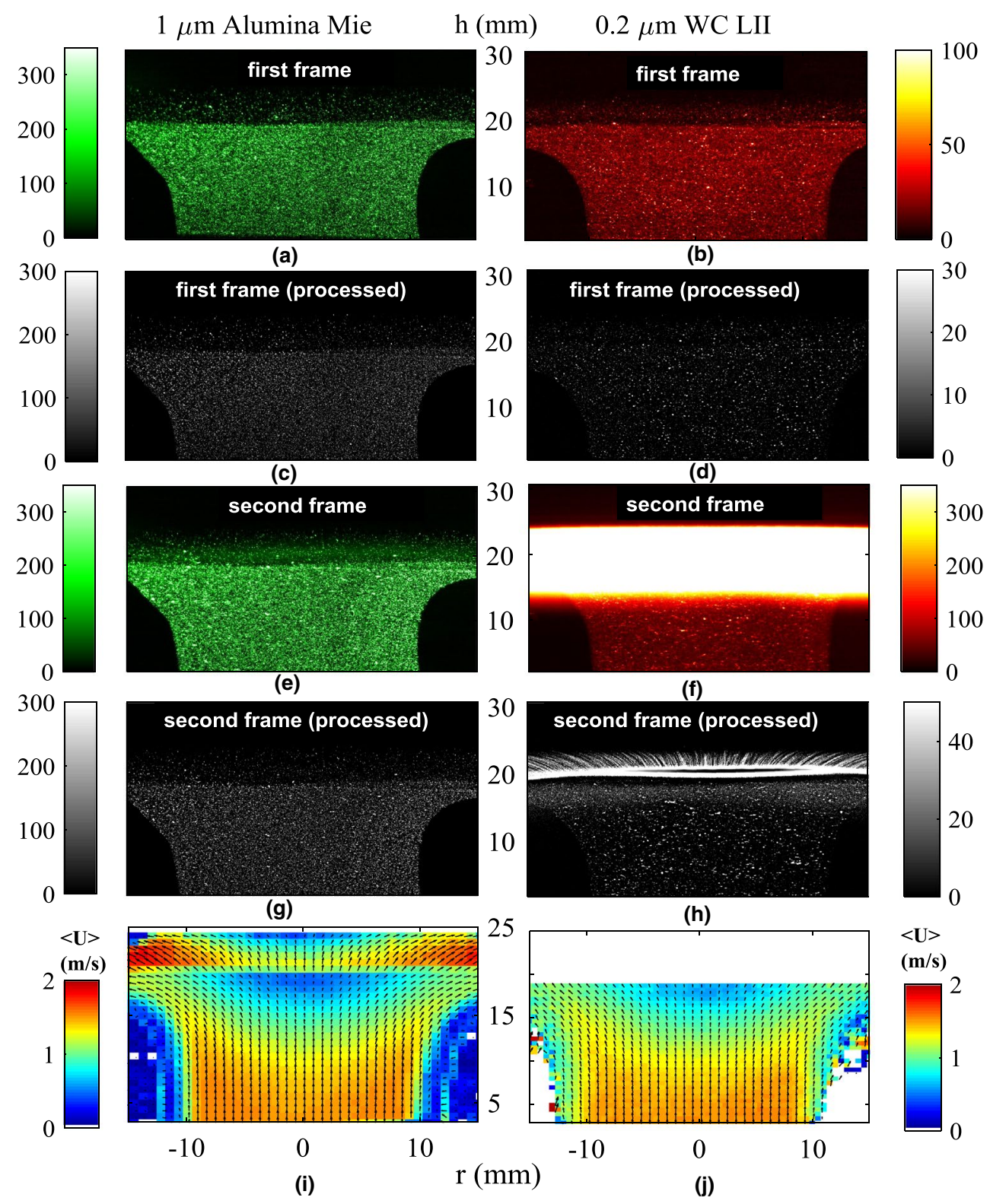

Fig. 14 a, b First frame a example PIV image; c, d preprocessed image (first frame); $\mathbf{e}, \mathbf{f}$ second frame of the PIV image; $\mathbf{g}, \mathbf{h}$ preprocessed image (second frame); $\mathbf{i}, \mathbf{j}$ mean velocity field. Left column is

based on Mie scattering from alumina tracer, and right column on LII signal from tungsten carbide particles, respectively

This is found to be the case for both alumina and WC tracers. Despite the drop in seeding density, there is still sufficient particle density in both Mie scattering and LII images downstream the flame front for a robust cross-correlation, which can be clearly seen after preprocessing the images (second row in Fig. 14). This shows a great advantage of LII-based PIV over the traditional color-based approach: if fluorescent particles were used for this case, the emission would no longer exist right downstream of the flame front due to thermal quenching and decomposition of the dopant.

However, on the second frame, the LII image becomes saturated by the flame luminosity (Fig. 14f), whilst the

flame only leaves a weak signature on the Mie-scatter image (Fig. 14e). Because of the readout mechanism of the low-speed CCD camera operated in the double-frame mode, the exposure time of the second frame is much longer than the first frame. As mentioned in Sect. 2.1, in the present case, the shutter time for the first frame is $5 \mu \mathrm{s}$, and $\sim 150 \mathrm{~ms}$ for the second frame, with an interframe interval of $5 \mathrm{~ns}$. This is a common problem for PIV measurements of luminous flames using a (low speed) CCD camera. Several solutions have been proposed to this problem: (a) using a narrow bandpass filter centered at the laser wavelength, as implemented in the present 
study for obtaining clean Mie-scatter images; (b) using a CMOS high-speed PIV camera, which has short exposure time for both frames; (c) reducing the gating time of the second frame with an extra shutter; and (d) using another CCD camera to record the particle images illuminated by the second laser pulse. Method (a) is not appropriate for the LII-PIV technique because black body radiation is also broadband, hence a narrow filter also reduces the strength of LII signal. Method (b) is feasible so long as the high-speed laser used has sufficient output energy to generate LII signals. Method (c) has previously been demonstrated by Li et al. (2008), in which high flame luminosity on the second frame was removed successfully using mechanical shutters. Since the shutters are not expensive, this is a good solution for low-speed LIIPIV in flames. Method (d) is simple and straightforward, but the two cameras need to be carefully aligned, and the background displacement needs to be measured with a static target and subtracted by image mapping during the preprocessing stage. Fig. 14i, j shows the measured velocity field based on the preprocessed images $(\mathrm{g}),(\mathrm{h})$. The LII-PIV method is capable of measuring the velocity upstream the flame front, however the vectors above $20 \mathrm{~cm}$ in height were lost owing to the overexposure problem. It is expected that with an extra shutter/camera, two-phase LII-PIV measurements can also be achieved on a particle-laden or droplet-laden flame successfully. A demonstration is currently underway implementing the LII-PIV technique in flames with the use of high frequency CMOS cameras.

\subsection{Possibility of applying LII-PIV to a sooty flame}

In Sect. 5.2, a premixed lean flame was used to demonstrate LII-PIV, where little soot was produced. If this technique was to be applied to a diffusion flame where large amounts of soot are produced, such as in Nordström et al. (2014), the LII signal from black tracers would be overwhelmed by the LII signal from soot particles, which can have a much higher volume fraction than the seeded tracers, and would have reached the sublimation temperature much earlier under the same laser fluence. However, the decay time of LII signal is proportional to the particle size, as larger particles need a longer time to cool down to the ambient temperature due to a smaller surface to volume ratio. The LII signal from soot particles usually lasts for several hundreds of nanoseconds, while the LII decay time of the tungsten carbide powder used in this study is between 1 and $2 \mu \mathrm{s}$. Hence, the LII signal from soot may be gated away, or at least reduced to a separable level, by carefully selecting the tracer size and using a proper gating strategy.

\section{Concluding remarks}

In this study, a novel PIV technique based on a LII signal from seeded submicron black particles is introduced to separately measure the velocity field corresponding to the liquid and gas phases of a two-phase flow. The black tracers are heated up to several thousand degrees to emit strong incandescence signal by a double-pulsed PIV laser running at high output energy, whilst the laser-induced temperature increase in large liquid droplets or solid particles is negligible. By collecting the LII signal at a wavelength different from the Mie scattering, the motion of seeded black tracers, which represents the gaseous flow field, can be separated from the motion of the disperse phase. Experimentally the LII-PIV technique was demonstrated on a single phase air jet, a droplet-laden flow, and finally a stagnation flame. The results were compared with those from a single-phase Miescatter PIV with $1 \mu \mathrm{m}$ alumina particles. The main conclusions are summarized as follows:

- Fine and inexpensive tungsten carbide (WC) tracer particles used in this study can accurately follow the gaseous flow, and the LII images are sufficiently robust to achieve accurate velocity measurements.

- LII-PIV is a low-cost but promising technique for measuring two-phase flows. In the droplet-laden flow case, by recording the Mie scattering from large droplets and the LII signal from WC particles separately, the liquid-phase and gas-phase velocities were measured independently. The LII-PIV technique is applicable to much higher droplet densities compared with previous methods.

- For the reacting case, the first frame of the LII image provides high quality particle images for cross-correlation even at the reaction and post-flame zone, whereas fluorescent or phosphorescent particles may not produce a detectable signal. However, the second frame is saturated by the flame luminosity, due to the long exposure times created in low-speed PIV. This problem can be solved by applying an extra shutter to reduce the shutter time of the second frame, as demonstrated by previous research, or by a gated or CMOS camera.

- For reacting flows, the density should be carefully controlled to avoid severe local gas heating and radiative heat loss. A seeding rate at the level of $10^{11}$ particles $/ \mathrm{m}^{3}$ is sufficient for velocity measurement whilst not disturbing the flame.

Future work focuses on demonstrating the LII-PIV technique on a two-phase flame (droplet-laden and particle-laden), and on sooty diffusion flames.

Acknowledgements The authors gratefully acknowledge the financial support from Universiti Teknologi Malaysia, and the funding by The 
Royal Society, Malaysian Industry-Government Group for High Technology and The Academy of Sciences Malaysia under the NewtonUngku Omar Fund: Advanced Fellowship (NA160115). The authors also acknowledge A.L.M.T. Corp (Japan) for providing tungsten carbide samples and relevant data.

Open Access This article is distributed under the terms of the Creative Commons Attribution 4.0 International License (http://creativeco mmons.org/licenses/by/4.0/), which permits unrestricted use, distribution, and reproduction in any medium, provided you give appropriate credit to the original author(s) and the source, provide a link to the Creative Commons license, and indicate if changes were made.

\section{References}

Balusamy S, Kamal MM, Lowe SM, Tian B, Gao Y, Hochgreb S (2015) Laser diagnostics of pulverized coal combustion in $\mathrm{O}_{2} / \mathrm{N}_{2}$ and $\mathrm{O}_{2} / \mathrm{CO}_{2}$ conditions: velocity and scalar field measurements. Exp Fluids 56:1-16

Cignoli F, Bellomunno C, Maffi S, Zizak G (2009) Laser-induced incandescence of titania nanoparticles synthesized in a flame. Appl Phys B 96:593-599

Dankers S, Schraml S, Will S, Leipertz A (2002) Application of laserinduced incandescence for the determination of primary particle sizes of nanoparticles demonstrated using carbon blacks. Chem Eng Technol 25:1160-1164

Driscoll KD, Sick V, Gray C (2003) Simultaneous air/fuel-phase PIV measurements in a dense fuel spray. Exp Fluids 35:112-115

Fan L, Gao Y, Hayakawa A, Hochgreb S (2016) temperature and velocity measurements by thermographic particle image velocimetry with $\mathrm{ZnO}$ tracers. Exp Fluids 58:1-34

Hassan YA, Blanchat TK, Seeley CH, Canaan RE (1992) Simultaneous velocity measurements of both components of a two-phase flow using particle image velocimetry. Int J Multiph Flow 18:371-395

Julien P, Whiteley S, Soo M, Goroshin S, Frost DL, Bergthorson JM (2015) Flame speed measurements in aluminum suspensions using a counterflow burner. Proc Combust Inst 000:1-8

Kariuki J, Mastorakos E (2017) Experimental investigation of turbulent flames in uniform dispersions of ethanol droplets. Combust Flame 179:95-116

Khalitov DA, Longmire EK (2002) Simultaneous two-phase PIV by two-parameter phase discrimination. Exp Fluids 32:252-268
Kiger KT, Pan C (2000) PIV technique for the simultaneous measurement of dilute two-phase flows. J Fluids Eng 122:811

Kock BF, Tribalet B, Schulz C, Roth P (2006) Two-color time-resolved LII applied to soot particle sizing in the cylinder of a diesel engine. Combust Flame 147:79-92

Kourmatzis A, Pham PX, Masri AR (2015) Characterization of atomization and combustion in moderately dense turbulent spray flames. Combust Flame 162:978-996

Krishnan SS, Lin K-C, Faeth GM (2001) Extinction and scattering properties of soot emitted from buoyant turbulent diffusion flames. J Heat Transf 123:331

Li Y-H, Wu C-Y, Chen B-C, Chao Y-C (2008) Measurements of a high-luminosity flame structure by a shuttered PIV system. Meas Sci Technol 19:045401

Michelsen H, Schulz C, Smallwood G, Will S (2015) Laser-induced incandescence: particulate diagnostics for combustion, atmospheric, and industrial applications. Progress Energy Combust Sci 51:1-47

Myers GD, Lefebvre AH (1986) Flame propagation in heterogeneous mixtures of fuel drops and air. Combust Flame 66:193-210

Nordström E, Olofsson NE, Simonsson J, Johnsson J, Bladh H, Bengtsson PE (2014) Local gas heating in sooting flames by heat transfer from laser-heated particles investigated using rotational CARS and LII. Proc Combust Inst 35:3707-3713

Petrosky BJ, Lowe KT, Danehy PM, Wohl CJ, Tiemsin PI (2015) Improvements in laser flare removal for particle image velocimetry using fluorescent dye-doped particles. Meas Sci Technol 26:115303

Sakakibara J, Wicker RB, Eaton JK (1996) Measurements of the particle-fluid velocity correlation and the extra dissipation in a round jet. Int J Multiph Flow 22:863-881

Sánchez AL, Urzay J, Liñán A (2015) The role of separation of scales in the description of spray combustion. Proc Combust Inst 35:1549-1577

Sipkens TA, Mansmann R, Daun KJ, Petermann N, Titantah JT, Karttunen M, Wiggers H, Dreier T, Schulz C (2013) In situ nanoparticle size measurements of gas-borne silicon nanoparticles by timeresolved laser-induced incandescence. Appl Phys B 116:623-636

Towers D, Towers C, Buckberry C, Reeves M (1999) A colour PIV system employing fluorescent particles for two-phase flow measurements. Meas Sci Technol 10:824-830 